http://jmscr.igmpublication.org/home/ ISSN (e)-2347-176x ISSN (p) 2455-0450

crossref DOI: https://dx.doi.org/10.18535/jmscr/v8i2.76

Journal Of Medical Science And Clinical Research

\title{
Comparative Study between IV Dexmedetomidine versus IV Dexmedetomidine with Nalbuphine as an Adjuvant for Monitored Anesthesia Care in Tympanoplasty Surgeries
}

\author{
Authors \\ Sowjanya Tungana ${ }^{1}$, A Satyanarayana ${ }^{2}$ \\ ${ }^{1}$ Post graduate student, Department of Anesthesiology, AMC, Visakhapatnam, Andhra Pradesh \\ ${ }^{2}$ Professor and HOD, Department of Anesthesiology, AMC, Visakhapatnam, Andhra Pradesh
}

\begin{abstract}
Background and Aims: Tympanoplasty surgeries are usually performed under Monitored Anaesthesia care (MAC).The selective a2 agonist dexmedetomidine, known for its opioid sparing effect along with sedative, analgesic hypotensive and anaesthetic properties with minimal respiratory depression has been used as a sole agent to provide MAC in various surgical interventions. The present study is aimed to evaluate the role of Dexmedetomidine as a sole sedoanalgesic agent and compare the efficacy of adding an adjuvant like Nalbuphine to Dexmedetomidine.

Material \& Methods: 100 patients of either sex, aged 18-20 years, ASA grade I\&II were randomized into two groups (D and ND) of 50 patients each for microscopic ear surgery under Local Anaesthesia (LA) with MAC. Group D received inj. Dexmedetomidine $1 \mathrm{mcg} / \mathrm{kg}$ iv loading dose while Group ND received inj nalbuphine $50 \mathrm{mcg} / \mathrm{kg}$ followed by Dexmedetomidine $1 \mathrm{mcg} / \mathrm{kg}$.Both groups received an infusion of inj. Dexmedetomidine @ $0.4 \mathrm{mcg} / \mathrm{kg} / \mathrm{hr}$. All patients were assessed for intraoperative haemodynamic changes, SpO2, Ramsay sedation score(RSS), and visual analogue scale(VAS).Rescue doses of sedatives, analgesics, satisfaction scores(Patients and Surgeons) were compared in both the groups. Data was analyzed using chi-square and t-test. p value $<0.05$ was considered significant.

Results: Mean Heart Rate (HR) and Mean Arterial Pressure (MAP) were significantly decreased from baseline in group ND as compared to group D ( $<<0.001)$. RSS, in group ND was significantly higher as compared to group D throughout the surgery. Rescue sedation was given in 5 patients in group ND while in group $D, 20$ patients required additional sedation $(p<0.01)$. Rescue analgesic with iv fentanyl was administered in 8 patients and 42 patients respectively in groups $N D$ and D.Patient and surgeon satisfaction scores were also significantly higher in group ND vs group $D(p<0.001)$.

Conclusion: A combination of Dexmedetomidine with Nalbuphine as an adjuvant for Monitored Anaesthesia Care in microscopic ear surgery was found to provide superior sedoanalgesia.

Keywords: Monitored ansthesia care, Dexmeditomedine, Nalbuphine, Tympanoplasty.
\end{abstract}

\section{Introduction}

Many ophthalmic and ENT surgeries are done under Local anaesthesia. In middle ear surgeries, LA provides several advantages such as less bleeding, cost-effectiveness, postoperative analgesia, faster mobilisation of the patient, and the ability to test hearing intraoperatively ${ }^{1}$ however it has been reported to be associated with dizziness, claustrophobia, anxiety, earache $e^{2,3}$ 
causing movement and increased bleeding hampering comfort of surgeon and patient.

Hence a technique adopted and defined by The American Society of Anaesthesiologists (ASA). The monitored anaesthesia care (MAC) a planned anaesthesia procedure during which a diagnostic or therapeutic procedure is performed under local anaesthesia together with sedation and analgesia ${ }^{4}$. The essential elements and purposes of MAC include safe sedation, control of the patient anxiety and analgesia ${ }^{5}$ Monitored anaesthesia care usually involves the administration of drugs with anxiolytic, hypnotic, analgesic, and amnestic properties either alone or as a supplement to local or regional techniques. So, there is always a need to find out an anaesthetic drug that can be used with LA block with maximum benefit and with minimum side effects.

Several drugs like propofol, benzodiazepines, opioids, $\alpha 2$ agonists were used for sedation, hypnosis, analgesia in the surgery done under MAC to improve the patient and surgical comfort. $^{6,7}$

Dexmedetomidine is a centrally acting a 2 receptor agonist with conscious sedation and analgesia effect without causing respiratory depression. Dexmedetomidine also acts as sympatholytic and can attenuate the stress response to surgery, thereby maintaining haemodynamic stability ${ }^{8,9}$. It has been reported to reduce the opioid requirements during and after surgery significantly However rescue analgesic doses are still required if it is used as a sole agent in surgery conducted under MAC. So we planned to add an adjuvant like nalbuphine which is a highly effective opioid agonist antagonist. It is a short-acting and rapid clearance drug compared with other opioids. Nalbuphine is less likely to cause side effects such as pruritus, respiratory depression, urinary retention and excessive sedation.

The primary aim of our study was to evaluate the role of Dexmedetomidine as a solesedoanalgesic agent for middle ear surgery and compare the efficacy of nalbuphine as an adjuvant to
Dexmedetomidine. The secondary aim of the study was to compare the haemodynamic parameters between the two groups, requirement of rescue analgesics and sedatives along with satisfaction scores among surgeons and patients.

\section{Material and Methods}

After obtaining approval from the ethics committee and informed patient consent, 100 patients of either sex aged between 18-55years, ASA grade1,2 were enrolled in the study. Those belonging to ASA class 111 or IV. presence of comorbidities. 2nd or 3rd-degree heart block, renal and hepatic disease, presence of coagulopathies hypersensitivity to any of the drugs used in this study, pregnant and lactating women were excluded from the study. This study is designed as a randomised, cross-sectional comparative study. One hundred patients were randomly divided into two groups, 50 patients in the group, Dexmedetomidine group-(Group D), and 50 patients in Dexmeditomidine nalbuphine Group (ND).

\section{Preoperative Preparation}

All patients were visited and evaluated thoroughly on the day before surgery. During an anaesthetic checkup, a thorough evaluation of all the systems were undertaken. A written informed consent is obtained from all the patients. Pre anaesthetic preparation of patient included a period of overnight fasting day before surgery. An airway assessment was also carried during the preanaesthetic checkup period. Routine laboratory examination was conducted: Complete haemogram, bleeding time clotting time, FBS, Blood Urea, Serum Creatinineweredone.ECG and chest X-ray was done in patients above 40 years. Monitoring used to record SPO2, Mean arterial pressure (MAP) and Heart rate continuously. Anaesthesia machine with - oxygen source- nasal cannula, Drugs to treat Hypotension, Bradycardia, Equipment for intubation, Emergency drugs were ready before starting the procedure. 


\section{Procedure}

When the patient was brought to the operation theatre, preoperative recordings of oxygen saturation, blood pressure, heart rate, respiratory rate, and ECG were obtained. Patients were placed supine on the operating table with the head turned opposite to the ear to be operated. The IV line is secured with $20 \mathrm{G}$ cannula, IV fluids of ringer lactate solution are started at a rate of $2 \mathrm{ml} / \mathrm{kg}$. Oxygen source was secured by nasal cannula at a rate of $2 \mathrm{lit} / \mathrm{min}$.

All patients in both groups were pre-medicated with IV inj. Glycopyrrolate.

Patient in Group D received bolus dose of Inj dexmedetomidine $1 \mathrm{mcg} / \mathrm{kg}$ over 10 minutes, followed by infusion $0.4 \mathrm{mcg} / \mathrm{kg} / \mathrm{hr}$. Patients in Group ND received Inj Nalbuphine $50 \mathrm{mcg} / \mathrm{kg}$ followed by bolus dose of Inj dexmeditomidine $1 \mathrm{mcg} / \mathrm{kg}$ followed by infusion of $0.4 \mathrm{mcg} / \mathrm{kg} / \mathrm{hr}$ IV.

Sedation level was assessed using the Ramsay sedation score. Sedation score titrated to $\geq 3$. Any patient RSS score is 6 procedure is converted to GA and excluded from the study. If the score was $<3$, rescue sedation dose with a bolus of midazolam $0.01 \mathrm{mg} / \mathrm{kg}$ was given. At the same time, the surgical area was prepared and draped. Then LA infiltration was given by operating surgeon using $2 \%$ lignocaine with adrenaline of 1:200,000 for blocking the tympanic branch of auriculotemporal nerve and great auricular nerve. (6-7ml)

Intraoperative pain was assessed using a visual analog scale (VAS). If the patient complained of pain with (VAS $\geq 3$ ) during the surgery, IV INJ Fentanyl was given as rescue analgesia, and even the surgeon used an additional dose of local anaesthetic infiltration of 2-3 $\mathrm{ml}$ with two \% lignocaine with adrenaline. The total number of patients receiving rescue sedatives and analgesia was noted.

Hemodynamic parameters, HR, MAP, SPO2, were recorded initially at $3 \mathrm{~min}, 6 \mathrm{~min}, 10 \mathrm{~min}$, and for every10 minutes till the end of surgery. Adverse effects of drugs like bradycardia is treated with atropine sulfate of $0.01 \mathrm{mg} / \mathrm{kg}$, and hypotension is treated with IV fluids and IV ephedrine hydrochloride $5 \mathrm{mg}$ in incremental doses. Any desaturation during the surgery is treated by increasing oxygen flow up to 6 litres, and up to 10 litres with bag and mask ventilation.

After completion of the surgery, postoperative pain assessed by VAS score if vas $>3$ rescue analgesia with Diclofenac was given. Patient and surgeon satisfaction score was assessed before shifting the patient from PACU to ward using 7point Likert verbal rating scale Acceptable satisfaction score of both the patient and surgeon being ${ }^{[5-7]}$.

\section{Monitoring}

Intraoperative monitoring of Heart rate using continuous Ecg. Mean arterial pressure, SPO2 observed and recorded every $3 \mathrm{~min}, 6 \mathrm{~min}, 10 \mathrm{~min}$ after giving loading dose, followed by every 10 min till the end of surgery.

Sedation score: The level of sedation was assessed using the Ramsay Sedation Score (RSS). The desired sedation level was defined as $\mathrm{RSS} \geq 3$.

- 1 = anxious, agitated, restless;

- 2 = cooperative, oriented, tranquil;

- 3 = responds to commands only;

- 4 = brisk response to a light glabellar tap or loud noise;

- 5 = sluggish response to a light glabellar tap or loud noise;

- $6=$ no response.

Analgesia Assessment: Analgesia of the patient assessed by Visual analog scale (VAS) 0-10.

- Where 0 indicated no pain,

- Five indicates distressing pain,

- Ten corresponds to maximum pain.

Patient Satisfaction Score: 7- point Likert scale verbal rating scale.

- $1=$ extremely dissatisfied

- 2 = dissatisfied;

- $3=$ somewhat dissatisfied

- 4 = undecided; 
- $5=$ somewhat satisfied;

- $6=$ satisfied

- $7=$ extremely satisfied.

Surgeon satisfaction score: 7 - point Likert scale verbal rating scale.

- 1 = extremely dissatisfied

- 2 = dissatisfied;

- $3=$ somewhat dissatisfied

- $4=$ undecided;

- $5=$ somewhat satisfied;

- $6=$ satisfied, $7=$ extremely satisfied.

\section{Statistical Methods}

- All the data was collected and was recorded in an excel sheet. Data were expressed in frequency and percentages when qualitative and in Mean SD when quantitative. A hundred patients were selected for the study. All of them underwent elective tympanoplasty and received the study drugs. No patient was dropped out of the study.

- Patient data were represented as mean and standard deviation (S.D) for continuous measurements, and categorical measurements are represented in number (\%). Significance was assessed at a 5\% level of significance.

- Student t-test (two-tailed, independent) has been used to find the significance of study parameters on a continuous scale between two groups.

- A Chi-square test has been used to find the significance of categorical measurements.

- In the present study, the statistical significance of sedation, analgesia and patient surgeon satisfaction score difference between group D (Dexmedetomidine) and group ND (Dex-Nalbuphinel) were analyzed. P-value <0.05 was taken to be statistically significant

\section{Observation and Results}

The demographic characteristics such as age, gender, weight were comparable in both groups $(\mathrm{P}>0.05)[\mathrm{Tab} 1]$.At the end of the loading dose of Dexmedetomidine, all patients reached RSS at 3 and none of the patients required additional supplementation of sedation at that time. Mean RSS was also significantly more in group ND as compared to group D throughout the surgery $(\mathrm{p}<0.001)$ [Tab2] Only 5 patients in group ND required rescue sedation in the form of iv midazolam single dose as compared to 20 patients in group D. $(\mathrm{p}<0.05)$ [Tab.3].10 patients in ND group and 40 patients in $\mathrm{D}$ group received additional dose of Local infiltration, Intraoperatively, 8 patients required rescue analgesia inj fentanyl in group ND and time to first rescue analgesia being at the mean of $85 \pm 10.85 \mathrm{~min}$, as compared to 42 patients in group D where it was given at the mean time of 38.33 \pm 13.59 min. $\quad(p<0.001)$. (Tab.4,5). 10 patients in group D had VAS $>5$ and they required post operative analgesia before shifting the patient to ward while none of the patient in group ND demanded analgesia postoperatively. The results were comparable in both the groups with regard to desaturation and none of the patients had fall of SpO2. Mean HR and MAP showed a significant fall from baseline in group ND as compared to patients in group D. $(\mathrm{P}<0.05)$.[Tab6,7][Fig1,2]. Patients satisfaction with regard to sedation and analgesia was higher in group ND than in group D $(P<0.0001)$ [Tab8].

Table1: Demographic variables

\begin{tabular}{|l|c|c|c|}
\hline Variables & GROUP ND & GROUP D & P-Value \\
\hline Age in Yrs & $33.3 \pm 8.4$ & $33.76 \pm 8.36$ & 0.785 \\
\hline Weight & $58 \pm 7.55$ & $57.78 \pm 7.37$ & 0.8 \\
\hline Sex $(\mathrm{M}: \mathrm{F})$ & $26: 24$ & $28: 22$ & 0.841 \\
\hline
\end{tabular}


Table 2: Showing Ramsay Sedation Score (RSS) \& Intraoperative Rescue Sedation

\begin{tabular}{|l|c|c|c|}
\hline \multirow{2}{*}{1. RSS } & Group ND & Group D & \multirow{2}{*}{ P Value } \\
\cline { 2 - 3 } & Mean + SD & Mean + SD & \\
\hline 10 Min. & $2.88+0.00$ & $3.00+0.01$ & 0.08 \\
\hline 20 Min. & $2.90+0.30$ & $2.71+0.68$ & $<0.001$ \\
\hline 30 Min. & $3.00+0.00$ & $2.60+0.49$ & $<0.001$ \\
\hline 40 Min. & $3.00+0.00$ & $2.50+0.61$ & $<0.001$ \\
\hline 60 Min. & $3.00+0.00$ & $2.32+0.43$ & 0.01 \\
\hline 90 Min. & $3.00+0.00$ & $2.50+0.46$ & $<0.001$ \\
\hline
\end{tabular}

Table 3: Distribution of study participants based on rescue sedation in Group ND \& Group D

\begin{tabular}{|l|c|c|c|c|}
\hline \multicolumn{2}{|l|}{} & GROUP ND & GROUP D & P VALUE \\
\hline $\begin{array}{l}\text { Number of Rescue } \\
\text { Sedation }\end{array}$ & YES & $5(10 \%)$ & $20(40 \%)$ & 0.032 \\
\cline { 2 - 4 } & NO & $45(90 \%)$ & $30(60 \%)$ & \multirow{2}{*}{ (Significant) } \\
\cline { 2 - 4 } & TOTAL & 50 & 50 & \\
\hline
\end{tabular}

Table 4: showing Visual Analogue Score (VAS) \& intraoperative Rescue analgesia

\begin{tabular}{|l|c|c|c|}
\hline & Group ND & Group D & \\
\cline { 2 - 3 } VAS Score & Mean + SD & Mean + SD & P Value \\
\hline 10 Min. & $2.10+1.06$ & $2.21+0.50$ & 0.5 \\
\hline 20 Min. & $2.32+1.24$ & $2.84+0.43$ & 0.006 \\
\hline 30 Min. & $2.57+1.29$ & $3.03+0.58$ & 0.02 \\
\hline 40 Min. & $3.15+1.05$ & $3.60+0.70$ & 0.01 \\
\hline 60 Min. & $3.38+1.16$ & $3.85+0.78$ & 0.01 \\
\hline 90 Min. & $3.58+1.21$ & $4.01+0.81$ & 0.03 \\
\hline
\end{tabular}

Table 5: Distribution of study participants based on rescue analgesia in Group D, ND.

\begin{tabular}{|l|l|c|c|c|}
\hline \multicolumn{2}{|l|}{ Variable } & GROUP ND & GROUP D & P VALUE \\
\hline Number of Rescue Analgesia (fentanyl) & YES & $8(16 \%)$ & $42(84 \%)$ & \multirow{2}{*}{ P $<0.0001$} \\
\cline { 2 - 4 } & NO & 42 & 8 & \\
\hline \multirow{2}{*}{ Number of Rescue Local Infiltration } & YES & $10(20 \%)$ & $40(80 \%)$ & \multirow{2}{*}{$\mathrm{P}<0.0001$} \\
\cline { 2 - 4 } & NO & $40(80 \%)$ & $10(20 \%)$ & \\
\hline
\end{tabular}

Table 6: Distribution of Mean heart rate in both groups at different time intervals

\begin{tabular}{|l|c|c|c|c|c|}
\hline \multirow{2}{*}{ Time } & \multicolumn{2}{|c|}{ GROUP ND } & \multicolumn{2}{c|}{ GROUP D } & \multirow{2}{*}{ P-Value } \\
\cline { 2 - 5 } & Mean & SD & Mean & SD & \\
\hline 3 & 66 & 5.14 & 70 & 5.20 & $0.0001^{* * *}$ \\
\hline 6 & 70 & 4.63 & 74 & 2.85 & $0.0108^{*}$ \\
\hline 10 & 75 & 5.68 & 76 & 2.28 & $0.0001^{* * *}$ \\
\hline 20 & 70 & 4.42 & 77 & 3.92 & 0.0001 \\
\hline 30 & 67 & 3.21 & 77 & 5.48 & 0.0001 \\
\hline 40 & 65 & 2.74 & 77 & 3.80 & 0.0001 \\
\hline 50 & 65 & 2.55 & 81 & 4.44 & 0.0001 \\
\hline 60 & 66 & 2.81 & 82 & 1.95 & 0.0001 \\
\hline.$/ 1 ; 70$ & 70 & 3.90 & 84 & 3.11 & 0.0001 \\
\hline 80 & 72 & 4.37 & 82 & 2.85 & 0.0001 \\
\hline 90 & 83 & 6.33 & 90 & 4.35 & 0.0001 \\
\hline
\end{tabular}




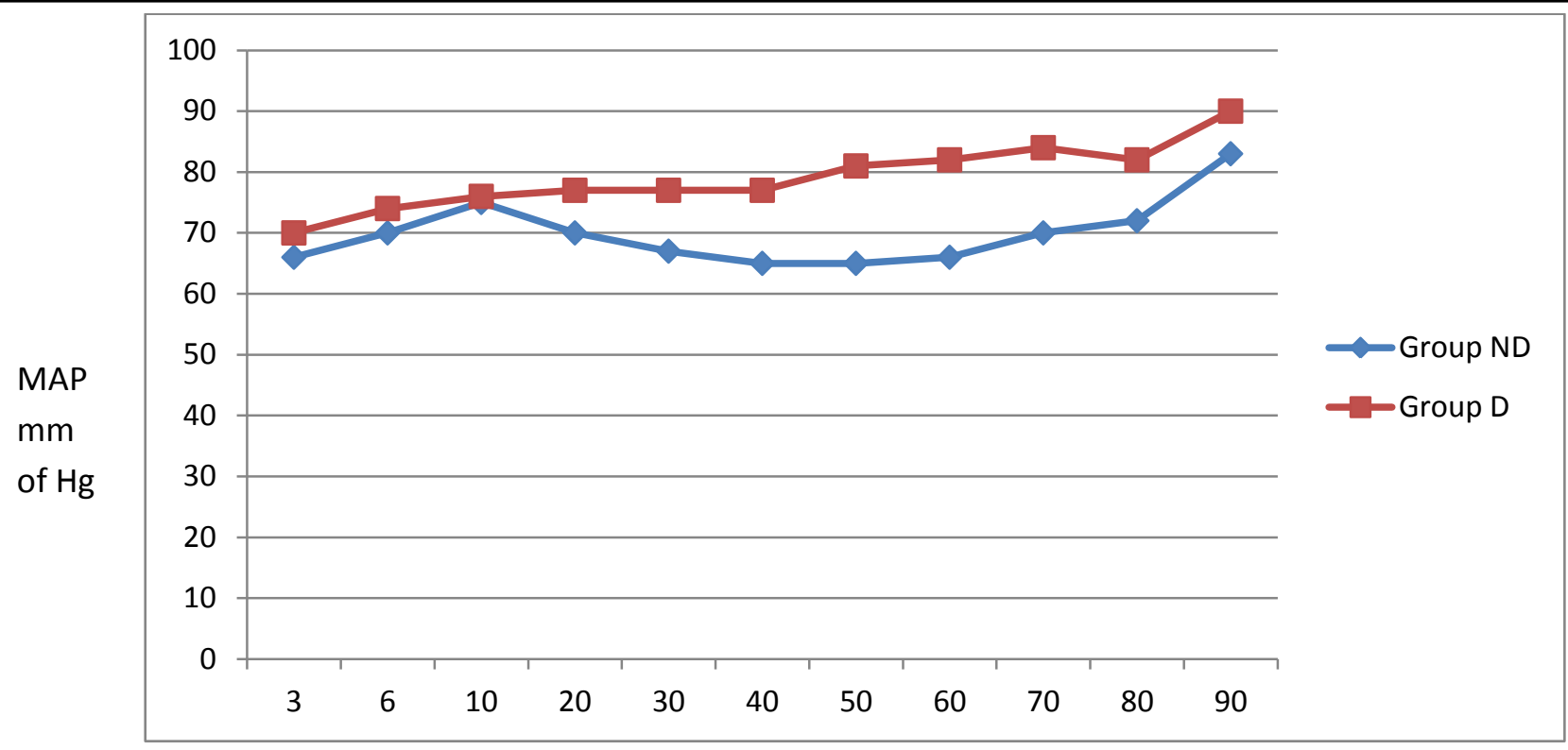

Time Scale

Fig 1 Distribution of Mean heart rate in both groups at different time intervals

Table 7: Distribution of Mean Arterial Blood Pressure in groups at different time intervals

\begin{tabular}{|l|c|c|c|c|c|}
\hline \multirow{2}{*}{ Time } & \multicolumn{2}{|c|}{ GROUP N D } & \multicolumn{2}{c|}{ GROUP D } & \multirow{2}{*}{ P-Value } \\
\cline { 2 - 5 } & Mean & SD & Mean & SD & \\
\hline 3 & 83 & 3.20 & 85 & 2.77 & $0.0012^{* * *}$ \\
\hline 6 & 88 & 3.67 & 90 & 2.06 & $0.001^{* *}$ \\
\hline 10 & 86 & 3.89 & 84 & 2.35 & $0.002^{* *}$ \\
\hline 20 & 76 & 3.19 & 83 & 2.16 & $0.0001^{* * *}$ \\
\hline 30 & 71 & 1.58 & 83 & 2.79 & 0.0001 \\
\hline 40 & 73 & 2.40 & 82 & 2.09 & 0.0001 \\
\hline 50 & 72 & 2.23 & 82 & 2.25 & 0.0001 \\
\hline 60 & 73 & 2.47 & 82 & 2.11 & 0.0001 \\
\hline 70 & 74 & 3.39 & 84 & 1.83 & 0.0001 \\
\hline 80 & 77 & 3.89 & 85 & 2.20 & 0.0001 \\
\hline 90 & 82 & 5.78 & 86 & 1.41 & 0.0001 \\
\hline
\end{tabular}

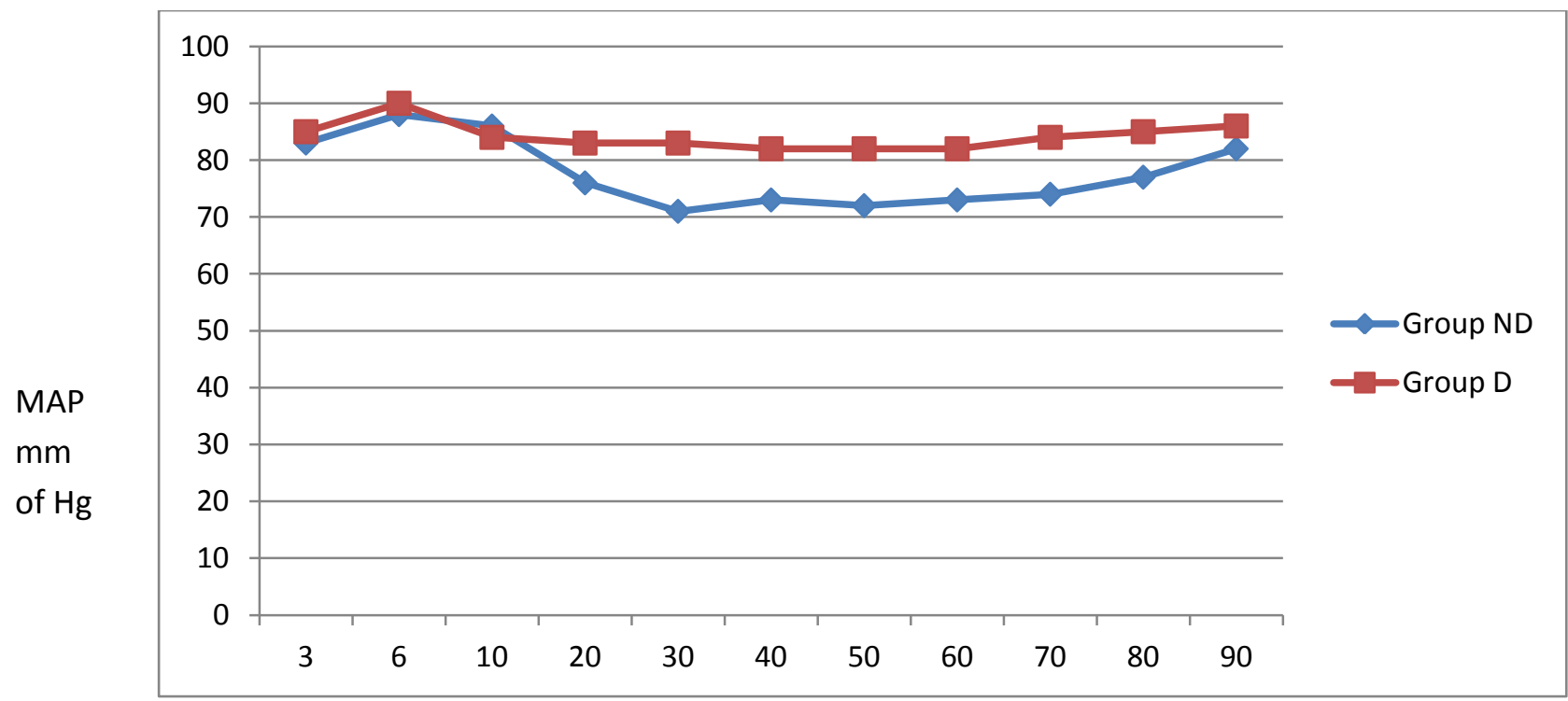

Time Scale

Fig 2: Distribution of Mean Arterial Blood Pressure in groups at different time intervals 
Table 8: Distribution of study participants based on Patient \& Surgeon Satisfaction score in Group N D \& Group D.

\begin{tabular}{|l|c|c|c|c|}
\hline \multicolumn{2}{|l|}{} & GROUP ND & GROUP D & P VALUE \\
\hline Patient Satisfaction score & 50 & 42 & 24 & 0.0003 (Significant)** \\
\hline Surgeon Satisfaction score & 50 & 39 & 26 & 0.0113 (Significant)** \\
\hline
\end{tabular}

\section{Discussion}

MESs are usually performed under MAC, in which an adequate sedation and analgesia without respiratory depression are desirable for comfort of both the surgeon and the patient. ${ }^{10}$

Parikh. DA et al ${ }^{11}$ compared dexmedetomidine as a sole agent against the traditional midazolamfentanyl combination in patients for Tympanoplasty under MAC and found qualitatively better sedation profile with dexmedetomidine. Verma.R et $\mathrm{al}^{12}$ compared dexmedetomidine with propofol for Tympanoplasty under LA and results suggested dexmedetomidine provided adequate sedoanalgesia without any adverse effects.

Combination of two drugs from the beginning of procedure allows the use of lower dose of each agent and thus decreases its undesired effects ${ }^{13}$. Synergism of Dexmedetomidine with other opioids decreases the need of complementary opioid analgesics. ${ }^{14}$ Alka Kewalramani, S.S. Jaitawat et al. ${ }^{15}$ compared Dexmedetomidine with Dexmedetomidine and Butorphanol as an adjuvant I.V. for MAC in Tympanoplasty and myringoplasty. They noted thatRSS was better in Dexmedetomidine with Butorphanol group which proves that Dexmedetomidine along with opioid provides better sedation which was our observation too. This study was aimed to assess the efficacy of adding adjuvant to dexmedetomidine, like Nalbuphine which is agonist antagonist opioid.

In our study we observed that intraoperative RSS was better in group ND Dexmedetomidine with Nalbuphine than group Dexmedetomidine throughout surgery. 5 patient from group ND required Inj.Midazolam as rescue sedation and 20 patients from group D required Inj. Midazolam, VAS score was lower in group ND than in group $\mathrm{D}$ with less requirement of rescue analgesic in group ND, Similar result was noted by Mahmoud Hassan Mohamed et al. ${ }^{16}$ who compared I.V. Dexmedetomidine and Nalbuphine with Midazolam and Nalbuphine in ear surgeries under MAC.Srinivasa Rao Nallam et $\mathrm{al}^{13}$.compared I.V.(Dexmedetomidine and Nalbuphine) with(Propofol and Nalbuphine) in patients undergoing middle ear surgeries. He also observed that Dexmedetomidine with Nalbuphine provides better sedation and analgesia in his study .

Our study demonstrated significantly higher patient and surgeon satisfaction score with Nalbuphine Dexmedetomidine combination.This suggests a difference in the quality of sedation and analgesia in both the groups.Dexmedetomidine also provides intense analgesia during postoperative period.dexmedetomidine nalbuphine have synergistic effect and none of the patient in this group complained of pain during the stay in PACU as compared to 10 patients in dexmedetomidine group who received diclofenac before shifting from PACU. The sedation and analgesic property of Dexmedetomidine is attributed to stimulation of $\alpha 2$ adrenoreceptor in locus coeruleus in the brain and modulation of transmission of nociceptive signals in CNS and at spinal level.

There was no statistically significant difference between the 2 groups regards saturation. Dexmedetomidine is unique in that it does not cause respiratory depression, because its effects are not mediated by the gamma amino butyric (GABA) system ${ }^{11}$

Reports of its use state that alpha 2 agonist effect is more specific but not alphaleffect (200:1 for clonidine \& 1600:1 for Dexmedetomidine), on administration of low and moderate doses and slow rates of infusion ${ }^{16}$. Consequently, peripheral vasoconstriction and hypertension would not be expected in these instances. Dexmedetomidine 
causes controlled hypotension \& thus provides better surgical (bloodless) field for microscopic surgery compared with other drugs like Midazolam, Propofol.

However in our study there was a significant decrease in HR and MAP from $20 \mathrm{~min}$. of the initial bolus which was subsequently maintained at significantly lower levels as compared to baseline values in group ND. This might be attributed to the sympatholytic, vagotonic and baroreflex sensitivity reducing effect of dexmedeto-midine. .Our findings are similar to other studies where lower HRand MAP were observed in the dexmedetomidinegroup. ${ }^{17}$ Hall JE et $a l^{18}$ compared the safety and efficacy of two doses (0.2 Vs $0.6 \mathrm{mcg} / \mathrm{kg} / \mathrm{hr}$ infusion) of Dexmedetomidine and found a $20 \%$ \& $16 \%$ decrease respectively of HR from baseline during the $10 \mathrm{~min}$. of initial dose.

Limitation of our study is that though BIS monitoring is more reliable parameter than RSS for monitoring sedation we used RSS due to unavailability of BIS.So it needs to be studied further by use of infusion of Dexmedetomidine along with BIS monitoring.

\section{Conclusion}

To conclude, the addition of Nalbuphine to Dexmedetomidine in Monitored Anaesthesia Care with local anaesthetics for middle ear surgery proves to be a better technique of MAC as compared to Dexmedeto-midine alone in terms of haemodynamic stability, intraoperative analgesia, sedation scores and satisfaction scores (surgeons and patients.)

\section{References}

1. Sarmento KM Jr., Tomita S. Retroauricular tympanoplasty and tympanomastoidectomy under local anesthesia and sedation. Acta Otolaryngol 2009;129:7268.

2. Caner G, Olgun L, Gültekin G, Aydar L. Local anesthesia for middle ear surgery. Otolaryngol Head Neck Surg
2005;133:295-7.

3. Yung MW. Local anaesthesia in middle ear surgery: Survey of patients and surgeons. Clin Otolaryngol Allied Sci 1996;21:4048.

4. Liang S, Irwin MG. "Review of anesthesia for middle ear surgery. Anesthesiol Clin 2010;28:519-28

5. Ghisi D, Fanelli A, Tosi M, Nuzzi M, Fanelli G.Monitored anesthesia care. Minerva Anestesiol 2005;71:533

6. Lee JJ, Lee JH. Middle-ear surgery under sedation: Comparison of midazolam alone or midazolam with remifentanil. J Laryngol Otol 2011;125:561-6.

7. Benedik J, Manohin A. Sedation for middle ear surgery:Prospective clinical trial comparing propofol and midazolam. Cent Eur J Med 2008;3:487-93.

8. Gupta K, Bansal M, Gupta PK, Pandey MN, Agarwal S.Dexmedetomidine infusion during middle ear surgery under general anaesthesia to provide oligaemic surgical field:A prospective study. Indian J Anaesth 2015;59:26- 30.

9. Sudheesh K, Harsoor S. Dexmedetomidine in anaesthesiapractice: A wonder drug? Indian J Anaesth 2011;55:323- 4.

10. Lee JJ, Lee JH. Middle- ear surgery under sedation: Comparison of midazolam alone or midazolam with remifentanil.J Laryngol Otol 2011;125:561- 6.

11. Parikh DA, Kolli SN, Karnik HS, Lele SS, Tendolkar BA. Aprospective randomized double blind study comparing Dexmedetomidine vs. combination of Midazolam Fentanylfor Tympanoplasty surgery under monitored anesthesia care. J Anaesthesiol Clic Pharmacol. 2013Apr; 29(2):1738.

12. Verma R, Gupta R, Bhatia VK, Bogra J, Agarwal SP. Dexmedetomidine and propofol for monitored anesthesia carein the middle ear surgery. Indian $\mathrm{J}$ Otol 2014;20:70- 4. 
13. Shrinivasa Rao Nallam et al Monitored anaesthesia care Comparison of Nalbuphine/ Dexmedetomidine versus Nalbuphine/Propofol for middle ear surgeries:A double blind randomized trial. November1, 2017, IP: 49.35.17.184.

14. De La Mora González JF, Robles Cervantes JA, Mora Martínez JM, Barba Alvarez F, Llontop Pisfil EdeL, González Ortiz M, Martínez Abundis E, Llamas Moreno JF, Espinel Bermúdez MC. Hemodynamic effects of dexmedetomidinefentanylvs. nalbuphinepropofolin plastic surgery. Middle East JAnaesthesiol. 2012 Feb;21(4):5537.

15. Alka Kewalramani(Chhabra) et al "Comparative Evaluation of IV Dexmedetomidine versus Dexmedetomidine with Butorphanol as an Adjuvant for Monitored Anaesthesia Care in Tympanoplasty and Myringoplasty: A Prospective, Controlled, Randomized, Double Blind Study" Indian Journal of Clinical Anaesthesia, JanuaryMarch,2016;3(1)

16. Mohamed MH, Hakim KK. Comparative study between dexmedetomidine/ nalbuphine and midazolam/nalbuphine in monitored anesthesia care during ear surgery. Egypt J Anaesth 2014;30:7-12.

17. Eren G, Cukurova Z, Demir G, Hergunsel O, Kozanhan B,Emir NS. Comparison of dexmedetomidine and three differentdoses of midazolam in preoperative sedation. $\mathrm{J}$ Anaesthesiol Clin Pharmacol 2011;27: 367- 72.

18. Hall JE, Uhrich TD, Barney JA, Arain SR, Ebert TJ. Sedative, amnestic, and analgesic properties of small-dose dexmedetomidine infusions. Anesth Analg 2000;90:699-705. 